\title{
BioéthiqueOnline
}

\section{Une politique concernant les données issues d'un programme de recherches interventionnelles en santé mondiale}

\author{
Valéry Ridde, Matthew Hunt, Christian Dagenais, Isabelle Agier, Aude Nikiema, \\ François Chiocchio, Emmanuel Bonnet et Paul-André Somé
}

Volume 5, 2016

URI : https://id.erudit.org/iderudit/1044267ar

DOI : https://doi.org/10.7202/1044267ar

Aller au sommaire du numéro

Éditeur(s)

BioéthiqueOnline

ISSN

1923-2799 (numérique)

Découvrir la revue

Citer cet article

Ridde, V., Hunt, M., Dagenais, C., Agier, I., Nikiema, A., Chiocchio, F., Bonnet, E. \& Somé, P.-A. (2016). Une politique concernant les données issues d'un programme de recherches interventionnelles en santé mondiale. BioéthiqueOnline, 5. https://doi.org/10.7202/1044267ar

\section{Résumé de l'article}

La recherche interventionnelle en santé mondiale est souvent effectuée au moyen de partenariats scientifiques internationaux. Mais la réalisation de telles recherches, notamment dans les pays à faible revenu où les enjeux de pouvoir, d'argent et de relations interculturelles sont exacerbés, soulève de nombreux défis éthiques, notamment concernant la conduite responsable lors des recherches collaboratives. L'un d'eux est la conservation, l'accès, l'utilisation et la publication des données. Malgré l'existence de multiples politiques et documents phares sur l'éthique et la conduite responsable de la recherche, les chercheurs en recherche interventionnelle sont encore peu outillés pour faire face à ce défi. Dans le contexte d'un programme de recherches interventionnelles réalisées au Burkina Faso, les auteurs souhaitent partager leur expérience dans le développement d'une politique interne concernant leurs données. Après avoir évoqué le contexte global et particulier, l'article présente le processus de formulation et d'adoption de cette politique par les chercheurs d'une équipe interdisciplinaire et internationale (Allemagne, Burkina Faso, Canada, France). Les valeurs qui enchâssent cette politique interne sont la transparence, la prévention, le consensus autour du processus de production, le respect des principes internationaux, l'accès libre aux connaissances produites et la préoccupation pour leur diffusion et utilisation. La politique se présente en plusieurs parties : objet, types et statuts des chercheurs impliqués, conservation des données, accès et utilisation des données, production scientifique et paternité. Les principaux défis rencontrés par les chercheurs pour formuler cette politique sont analysés dans un souci de partage pour susciter un débat rarement abordé. La confiance, la transparence et le respect mutuel doivent être au coeur de tout partenariat scientifique en recherche interventionnelle.
Droits d'auteur @ V Ridde, M Hunt, C Dagenais, I Agier, A Nikiema, F Chiocchio, E Bonnet et P-A Somé, 2016

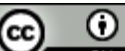

Ce document est protégé par la loi sur le droit d'auteur. L'utilisation des services d'Érudit (y compris la reproduction) est assujettie à sa politique d'utilisation que vous pouvez consulter en ligne.

https://apropos.erudit.org/fr/usagers/politique-dutilisation/ 


\title{
Une politique concernant les données issues d'un programme de recherches interventionnelles en santé mondiale
}

\author{
ARTICLE (RÉVISION PAR LES PAIRS / PEER-REVIEWED) \\ Valéry Ridde ${ }^{1-2}$, Matthew Hunt ${ }^{3}$, Christian Dagenais ${ }^{4}$, Isabelle Agier ${ }^{1-2}$, Aude Nikiema ${ }^{5}$, François Chiocchio ${ }^{6}$, \\ Emmanuel Bonnet ${ }^{7}$, Paul-André Somé ${ }^{8}$
}

An English version of this article appears in Annexe B; une version anglaise de cet article est disponible à l'Annexe B.

Reçu/Received: 2 Aug $2015 \quad$ Publié/Published: 29 Apr 2016
Éditrices/Editors: Vanessa Chenel \& Cécile Bensimon
Évaluateurs externes/Peer-Reviewers: François Alla \& Hubert Doucet

2016 V Ridde, M Hunt, C Dagenais, I Agier, A Nikiema, F Chiocchio, E Bonnet, P-A Somé, Creative Commons Attribution 4.0 International License

\section{Résumé}

La recherche interventionnelle en santé mondiale est souvent effectuée au moyen de partenariats scientifiques internationaux. Mais la réalisation de telles recherches, notamment dans les pays à faible revenu où les enjeux de pouvoir, d'argent et de relations interculturelles sont exacerbés, soulève de nombreux défis éthiques, notamment concernant la conduite responsable lors des recherches collaboratives. L'un d'eux est la conservation, l'accès, l'utilisation et la publication des données. Malgré l'existence de multiples politiques et documents phares sur l'éthique et la conduite responsable de la recherche, les chercheurs en recherche interventionnelle sont encore peu outillés pour faire face à ce défi. Dans le contexte d'un programme de recherches interventionnelles réalisées au Burkina Faso, les auteurs souhaitent partager leur expérience dans le développement d'une politique interne concernant leurs données. Après avoir évoqué le contexte global et particulier, l'article présente le processus de formulation et d'adoption de cette politique par les chercheurs d'une équipe interdisciplinaire et internationale (Allemagne, Burkina Faso, Canada, France). Les valeurs qui enchâssent cette politique interne sont la transparence, la prévention, le consensus autour du processus de production, le respect des principes internationaux, l'accès libre aux connaissances produites et la préoccupation pour leur diffusion et utilisation. La politique se présente en plusieurs parties: objet, types et statuts des chercheurs impliqués, conservation des données, accès et utilisation des données, production scientifique et paternité. Les principaux défis rencontrés par les chercheurs pour formuler cette politique sont analysés dans un souci de partage pour susciter un débat rarement abordé. La confiance, la transparence et le respect mutuel doivent être au cœur de tout partenariat scientifique en recherche interventionnelle.

\section{Mots clés}

recherche interventionnelle, donnée, santé mondiale, politique, intégrité, publication, paternité, conduite responsable de la recherche

\section{Abstract}

Interventional global health research is often carried out through international scientific partnerships. However, the conduct of such research, especially in low-income countries where issues of power, money and intercultural relations are exacerbated, raises many ethical challenges particularly related to responsible conduct in collaborative research. One of these challenges is the storage, access, use and publication of data. Despite the existence of multiple influential policies and documents on ethics and the responsible conduct of research, researchers in intervention research are still not equipped to deal with such challenges. In the context of an intervention research program conducted in Burkina Faso, the authors would like to share their experience in the development of an internal policy concerning their data. After presenting the global and the particular context, the paper presents the process of policy formulation and its adoption by the researchers of an interdisciplinary and international team (Burkina Faso, Canada, France, Germany). The values that entrench this internal policy are: transparency, prevention, consensus about the production process, respect for international principles, free access to the knowledge produced and concerns regarding its dissemination and use. The policy is presented in several parts: aim, types and status of the participating researchers, data storage, access and use of data, scientific production and authorship. The main challenges faced by researchers in formulating this policy are analyzed in a shared desire to spark a debate that is rarely addressed. Trust, transparency and mutual respect should be central to any scientific partnership in intervention research.

\section{Keywords}

intervention research, data, global health, policy, integrity, publication, authorship, responsible conduct of research 


\section{Responsabilités des évaluateurs externes}

Les évaluations des examinateurs externes sont prises en considération de façon sérieuse par les éditeurs et les auteurs dans la préparation des manuscrits pour publication. Toutefois, être nommé comme examinateur n'indique pas nécessairement l'approbation de ce manuscrit. Les éditeurs de BioéthiqueOnline assument la responsabilité entière de l'acceptation finale et la publication d'un article.
Peer-reviewer responsibilities

Reviewer evaluations are given serious consideration by the editors and authors in the preparation of manuscripts for publication. Nonetheless, being named as a reviewer does not necessarily denote approval of a manuscript; the editors of BioéthiqueOnline take full responsibility for final acceptance and publication of an article.

Affiliations des auteurs / Author Affiliations

${ }^{1}$ Département de médecine sociale et préventive, École de santé publique de l'Université de Montréal (ESPUM), Montréal, Canada

${ }^{2}$ Institut de recherche en santé publique de l'Université de Montréal (IRSPUM), Montréal, Canada

${ }^{3}$ School of Physical and Occupational Therapy, McGill University, Montreal, Canada

${ }^{4}$ Département de psychologie Université de Montréal, Montréal, Canada

${ }^{5}$ Institut des Sciences des Sociétés (INSS), CNRST, Ouagadougou, Burkina Faso

${ }^{6}$ École de gestion TELFER, Université d'Ottawa, Ottawa, Canada

${ }^{7}$ Identités et Différenciations de l'Environnement des Espaces et des Sociétés - Caen (IDEES CNRS), Université de Caen Basse-Normandie; IRD - UMI RESILIENCES 236, Caen, France

${ }^{8}$ Action-Gouvernance-Intégration-Renforcement; Groupe de travail en Santé et Développement (AGIR/SD),

Ouagadougou, Burkina Faso

\section{Correspondance / Correspondence}

Valéry Ridde, valery.ridde@umontreal.ca

\section{Remerciements}

Nous tenons à remercier tous les chercheurs membres de l'équipe qui ont bien voulu commenter le contenu de la politique ainsi que Alice Desclaux et Jill Murphy pour leurs relectures et conseils de lectures pour formuler la politique. Nous remercions aussi les deux évaluateurs de l'article dont les commentaires ont permis de renforcer nos arguments. Le programme de recherche dont il est question dans cet article a bénéficié d'une subvention de recherche des Instituts de recherche en santé du Canada (IRSC), \#ROH-115213. Valery Ridde est titulaire d'une Chaire de recherche en santé publique appliquée des IRSC, CPP 137901. Matthew Hunt est chercheur boursier (junior 1) des Fonds de recherche du Québec-santé.

\section{Conflit d'intérêts}

Valery Ridde et Matthew Hunt sont des collègues et collaborent avec Bryn Williams-Jones, l'éditeur-en-chef de BioéthiqueOnline. Ce dernier n'a participé à aucun moment au processus d'évaluation ou à l'examen par les pairs de cet article.

\section{Acknowledgements}

We would like to thank all researchers that are members of the team who took time to comment on the content of the policy as well as Alice Desclaux and Jill Murphy for their proofreading and reading suggestions for formulating the policy. We also thank the two reviewers of the article whose comments contributed to strengthen our arguments. The research program referred to in this article received a grant from the Canadian Institutes of Health Research (CIHR), \#ROH-115213. Valery Ridde holds a CIHR Research Chair in Applied Public Health, PPC 137901. Matthew Hunt is a Research Fellow (junior 1) of the Québec Research FundHealth.

\section{Conflicts of Interest}

Valery Ridde and Matthew Hunt are colleagues of and collaborate with Bryn Williams-Jones, the Editor-in-chief of BioéthiqueOnline. The Editor-in-chief was not involved in any part of the evaluation or peer-review process of this article.

\section{Introduction}

Alors que la recherche interventionnelle en santé publique est en plein développement, sa réalisation dans un contexte de santé mondiale, notamment en Afrique où les enjeux de pouvoir et d'argent sont consubstantiels à la collaboration internationale [1,2], pose des défis éthiques majeurs concernant les données et leur publication. Pour y faire face, notre équipe a développé une politique interne concernant la conservation, l'accès, l'utilisation et la publication de ces données. Dans cet article, nous souhaitons présenter ce processus de développement pour partager notre expérience et susciter un débat a priori essentiel à une conduite responsable de la recherche [3,4]. II s'agira moins de partager des enjeux traditionnels de l'éthique et de la protection des personnes ou des participants 
aux recherches que des défis de la collaboration internationale, de la déontologie des chercheurs et de leur pratique de recherche. En outre, notre réflexion s'inscrit dans ce qui est aujourd'hui (re)nommée la recherche interventionnelle en santé publique puisque l'expérience que nous décrivons s'inscrit dans un programme d'analyses d'interventions mis en œuvre au Burkina Faso, dont nous détaillons les contours plus bas.

Dans cet article, nous présentons d'abord le contexte de la conduite des recherches interventionnelles en santé mondiale pour aborder, ensuite, la question de la conduite responsable de la part des chercheurs. Puis, nous abordons le contexte particulier de notre programme de recherches qui a donné lieu à la définition d'une politique interne concernant les données, dont nous analysons le processus de construction et ses défis pour terminer l'article.

\section{Les défis de la conduite des recherches interventionnelles en santé mondiale}

Si la recherche interventionnelle en santé publique (RISP) n'est pas nouvelle, on assiste depuis quelques années à un renouveau pour mieux en définir le champ, les disciplines, et les compétences des chercheurs [5,6]. Sans retourner à l'étude de son histoire, on définira la RISP comme étant la mobilisation d'une démarche scientifique pour l'analyse des interventions de santé populationnelles. Le développement de la RISP a pris aujourd'hui une ampleur relativement importante au Canada, notamment grâce à l'impulsion donnée par les Instituts de Recherche en Santé du Canada (IRSC) et son Institut de la santé publique et des populations, à l'image de ce qui se déroule en Europe $[7,8]$ ou en Australie [9], par exemple. Cependant, ce mouvement semble encore timide dans les pays à faible et moyen revenu, et notamment en Afrique où la RISP reste rare [10]. Les études épidémiologiques sont toujours prépondérantes sur le continent africain et les analyses des interventions complexes, au moyen d'une approche interdisciplinaire et de méthodes mixtes sont encore inhabituelles [11] alors qu'elles sont pourtant consubstantielles à la RISP.

Parmi les compétences importantes que les chercheurs doivent maitriser, le groupe de travail de I'Initiative de recherche interventionnelle en santé des populations du Canada (IRISPC) propose d'appliquer " un ensemble pertinent de principes éthiques au processus de recherche " [12]. II précise notamment la nécessaire posture réflexive que les chercheurs doivent mobiliser, concernant tant leurs comportements que leurs compétences ou les effets de leurs actions. À ce titre, certains considèrent que "science » et "éthique » sont inséparables, s'influencent réciproquement et amènent les chercheurs à adopter des postures normatives évolutives [13]. Cependant, le groupe n'est pas allé beaucoup plus loin, n'a pas défini précisément ces principes, laissant certainement libre cours aux différentes équipes le soin de les développer dans leur contexte particulier, ce qui est justement l'objet de notre article.

Les questions éthiques du développement de la RISP en santé mondiale doivent être prises au sérieux [14-16]. Cette problématique particulière, au cœur de cet article, concerne moins les discussions traditionnelles de la bioéthique (autonomie, justice, bienfaisance, non-malfaisance, protection des personnes, etc.) que la conduite des recherches et notamment, le comportement des chercheurs, évidemment influencé par leurs institutions, au sens large du terme. On ne s'attardera pas non plus sur les enjeux éthiques associés aux interventions [17], en tant que telles, puisque la RISP, contrairement à la recherche-action, fait en sorte que les chercheurs ont souvent une position plus distanciée et ils agissent peu sur le contenu et la mise en œuvre des actions. Ainsi, compte tenu de la complexité inhérente à l'objet de la RISP, les chercheurs doivent aussi être en mesure de travailler en équipe, en collaboration avec des collègues provenant d'autres disciplines et d'autres cultures organisationnelles, avec des intervenants et des décideurs réclamant que les résultats des recherches soient utiles et utilisables [18]. Dans ce contexte, ces défis se cristallisent autour, notamment, de trois principaux types de collaborations scientifiques associées à la RISP : disciplinaires, géographiques, organisationnelles [19]. Ces différents enjeux soulèvent à leur tour des 
défis de nature éthique, que nous explicitons par la suite, concernant notamment l'interdisciplinarité, le travail en équipe et l'application des connaissances. Ils paraissent relativement exacerbés en ce qui concerne la recherche en santé mondiale.

\section{La conduite responsable en contexte de collaboration internationale}

Nos travaux précédents, et bien d'autres, ont montré combien les questions de pouvoir et d'argent pouvaient influencer ces processus de recherche en équipe, notamment dans un contexte de relations Nord-Sud, d'histoire coloniale et de dépendance financière [20-22]. Par exemple, au Burkina Faso, $87 \%$ des projets de recherche en santé sont financés par des ressources extérieures [23]. Les cultures organisationnelles et individuelles dans le domaine de la RISP peuvent être très différentes d'un pays ou d'un continent à l'autre. Les partenariats scientifiques, inhérents à cette forme de pratique de recherche en santé publique, sont complexes à mobiliser [1] et font ressortir de nombreux enjeux éthiques [24]. Ils concernent, par exemple, la définition des questions de recherche et leur alignement avec les priorités des pays, la répartition et la gestion des ressources financières, les processus de prise de décision, la propriété des données collectées, etc. Nous pourrions aussi évoquer le double fardeau, et ses implications, posé par ce type de collaboration scientifique impliquant la nécessaire participation des comités d'éthique de la recherche du Nord et du Sud [25]. De surcroît, les membres des comités d'éthique de la recherche qui analysent les protocoles sont souvent peu formés à analyser les RISP, peu disponibles et ne disposent pas de tous les moyens nécessaires pour exercer correctement leur rôle [26].

Dans le domaine des publications et de la signature scientifiques, les défis éthiques dans le contexte de ces collaborations internationales sont immenses, mais peu étudiés [27]. Le fait que la plupart des premiers auteurs des articles tirés de ces partenariats soient situés dans des institutions du Nord est source de débat [28]. Notre analyse des articles parus depuis 30 ans concernant le financement de la santé au Burkina Faso montre que les chercheurs du pays ne sont premiers auteurs que dans $5 \%$ des articles auxquels ils participent [29]. Cette question devient importante puisque la collaboration scientifique internationale est en croissance. Par exemple, la proportion d'articles toutes disciplines confondues où les coauteurs sont issus de pays différents est passée de $16 \%$ à $25 \%$ entre 1997 et 2012 à l'échelle mondiale, de même que le nombre d'auteurs par article ne cesse d'augmenter [30]. Dans le domaine des sciences naturelles et de la santé, si $87 \%$ des articles en 1900 comportaient un seul auteur, ce n'est plus le cas que pour $7 \%$ en 2011 [31]. De plus, la question de l'accès aux données apparait très sensible, car bien souvent, les protocoles sont écrits au Nord avec très peu d'implication des chercheurs du Sud (pour de multiples raisons que nous ne pouvons ici évoquer faute de place, voir [21]), les données sont collectées par les collègues du Sud puis analysées par ceux du Nord. Cette représentation est certes caricaturale, mais représente bien souvent la réalité évoquée $[20,32]$.

En outre, dans un contexte où les chercheurs africains sont souvent accaparés par des consultations internationales [22], ce sont habituellement de jeunes chercheurs, des étudiants ou des assistants qui sont au cœur des processus de recherche, de collecte et d'analyse des données. Au Nord, ces collaborations scientifiques en santé mondiale impliquent aussi souvent des étudiants (venant aussi du Sud) dont l'implication permet le renforcement de leur capacité de recherche. Ainsi, les enjeux de formation de la relève s'ajoutent à l'ensemble de ces enjeux et se posent de manière cruciale [33]. Cela est d'autant plus important que les défis éthiques commencent à peine à être abordés dans les formations doctorales de santé publique au Canada et sont quasi-inexistantes en Afrique.

De ce fait, de nombreux chercheurs qui ont été impliqués dans ce type de collaboration ont parfois constaté des écarts de conduite, des manquements aux règles d'éthique et d'utilisation des données [34]. Ces écarts ne sont évidemment pas le propre des chercheurs ou étudiants travaillant en Afrique, ils existent partout [35]. Nous pensons cependant que le terrain de la RISP dans un contexte de collaboration en santé mondiale décrit précédemment peut exacerber ces dérives. Quel 
chercheur de santé mondiale n'a pas entendu l'histoire de ce professeur de médecine fournissant des données issues d'un projet de recherche international et à l'insu de ses partenaires, à son étudiant pour qu'il puisse écrire sa thèse? Qui n'a pas été surpris de voir apparaitre dans la liste des auteurs, le nom d'un chercheur n'ayant contribué d'aucune manière au contenu et à l'écriture d'un article scientifique? À l'inverse, comment ne pas être surpris de voir un chercheur, ayant contribué significativement à une étude, oublié dans la signature scientifique d'un article ou même dans les remerciements? Qui ne s'est pas étonné que des chercheurs s'évertuent à collecter des données pendant des années, mais ne les publient pas, ou trop tard pour que les résultats soient utiles aux intervenants, ou ne s'attachent pas à les rendre intelligibles pour les utilisateurs potentiels et ne considèrent pas cela comme un problème éthique?

Nous pourrions multiplier les exemples pendant de nombreuses pages et tenter d'en expliquer les raisons, mais ce n'est pas l'objectif de cet article. En effet, nous souhaitons plutôt faire progresser la réflexion sur ces enjeux touchant la conduite des chercheurs et concernant l'accès et l'utilisation des données issues de projets de RISP en santé mondiale. Ainsi, l'objectif de cet article est de décrire le contexte et le processus de réalisation d'une politique concernant la conservation, l'accès et l'utilisation de données empiriques produites dans le cadre d'un programme de recherches en santé mondiale. Nous pensons que le partage de ce processus, ainsi que le contenu de cette politique, permettront de susciter un débat entre chercheurs et éventuellement de soutenir d'autres équipes.

\section{Contexte et processus de formulation de la politique interne}

Le contexte de cette politique interne est celui de la RISP et plus spécifiquement en Afrique, dans le cadre d'une collaboration scientifique internationale entre de multiples institutions de recherche (publiques et privées) d'Allemagne, du Burkina Faso, du Canada et de France. Cette politique a été formulée pour un programme de recherche réalisé au Burkina Faso, financé pour cinq ans (20112016) par les IRSC dans le cadre de son appel pour des recherches programmatiques répondant à l'équité et la santé. Le concept de recherche programmatique suppose que les chercheurs présentent un programme proposant de multiples recherches, qui peuvent évoluer dans le temps, mais qui doivent toutes concourir à un objectif commun. Dans le cas présent, cet objectif concerne l'équité et la santé en associant un groupe de chercheurs à des décideurs.

Notre recherche programmatique consiste en un portefeuille de RISP afin d'évaluer l'impact, l'efficacité et les processus d'interventions communautaires prometteuses pour améliorer l'équité au Burkina Faso. Ces interventions concernent la santé maternelle et infantile, la santé des indigents, les maladies fébriles, les traumatismes liés aux accidents de la route, les réformes des systèmes de santé. Pour favoriser le partage et l'utilisation des résultats, une intervention de courtage en connaissance a été formulée, mise en œuvre et évaluée. Plusieurs recherches-actions ont été mise en œuvre et certains développements méthodologiques analysés. L'ensemble des détails de ce programme, de ses réalisations et des partenaires est présenté ailleurs (voir Équité en santé au Burkina Faso). Le budget total du programme est de CDN\$1,8 million.

Le processus de formulation de cette politique s'est déroulé par étapes, que nous décrivons plus bas, sous la coordination du chercheur principal (CP), premier auteur de cet article (VR), et avec l'implication de certains chercheurs associés au programme et coauteurs de cet article. II a été entrepris à la fin de l'année 2014, environ deux ans après le démarrage du programme. Le CP a pris le leadership de la production et de la coordination de ce processus, car il en avait l'initiative et la responsabilité morale, avait déjà étudié la question dans des travaux préalables et était le seul à disposer d'une vision d'ensemble des projets de RISP intégrés dans ce programme. Cependant, le processus a été inclusif et participatif, chacun ayant eu l'opportunité d'apporter ses compétences et expériences. 
Le contexte et les analyses des défis de la collaboration scientifique en santé mondiale étant bien connus par le CP [20,21]. II a amorcé cette réflexion, dans une première étape, par une analyse des écrits sur le sujet. Une revue non systématique des écrits a donc été entreprise pour en permettre une analyse narrative. Les principaux articles scientifiques sur le sujet, et notamment ceux cités plus haut, ont été étudiés ainsi que les documents et rapports concernant la conduite responsable de la recherche. Cependant, au-delà des grands principes et guides concernant la pratique de recherche, il faut bien reconnaître que rares sont les chercheurs qui publient de tels processus, et encore plus rares sont les politiques sur les données empiriques accessibles sur Internet. Dans une seconde étape, il a donc fallu innover en rédigeant un document abordant les différents points essentiels et en s'inspirant notamment des principes issus des textes importants sur le sujet au Canada et au Québec (Encadré 1). En outre, une longue série de documents provenant de plusieurs pays concernant ce sujet ont été étudiés et sont présentés en annexe 4 de la politique (Annexe A).

\section{Encadré 1 : documents phares sur l'éthique et la conduite responsable de la recherche}

- La Politique des trois organismes (IRSC, CRSNG, CRSH) sur le libre accès aux publications, 2015

- La Politique sur la conduite responsable en recherche des FRQ, 2014

- L'énoncé de politique des trois Conseils canadiens : éthique de la recherche avec des êtres humains, 2014

- Le cadre de référence des trois organismes (IRSC, CRSNG, CRSH) sur la conduite responsable de la recherche, 2011

- Le règlement en matière de probité intellectuelle en recherche et développement. CHUM, 2005

Ces textes fondateurs sont présentés en préambule de la politique interne pour s'assurer que les chercheurs impliqués puissent les repérer. Les chercheurs canadiens sont censés les connaître (bien que les formations soient rares à ce sujet) et les autres ont certainement appréhendé les principes dans des politiques similaires de leurs pays. Cependant, le document interne de politique vise surtout à contextualiser ces principes directeurs en une démarche opérationnelle spécifique à notre programme de recherche. Une première version de la politique interne a donc été élaborée par le CP sur la base de l'ensemble de ces documents et des besoins du programme de recherches au Burkina Faso.

Dans une troisième étape, elle a été acheminée à un premier groupe restreint des six chercheurs les plus proches du programme et des enjeux éthiques en recherche. Deux chercheurs externes au programme (du Canada et de France), mais disposant d'une expertise sur le sujet et d'une expérience en Afrique, ont aussi été sollicités pour obtenir des conseils éclairés sur l'ébauche du document. Les commentaires (non exhaustifs ici) ont notamment souligné le besoin de :

- préciser les enjeux liés à la publication en accès libre des articles

- préciser le concept de données utilisé dans la politique

- tenir compte de la spécificité de la recherche qualitative lorsque l'on évoque la transmission des données et leur caractère anonyme

- $\quad$ proposer un stockage électronique, mais aussi physique des données

- partager les programmes de nettoyage des données quantitatives

- préciser la notion de chercheur dans le contexte d'une subvention des IRSC et de multiples projets de recherche au sein d'un même programme en collaboration

- discuter de la congruence entre les principes de la politique interne et celles des comités d'éthiques de la recherche où les projets ont été soumis

- distinguer entre les personnes qui réalisent les analyses et celles qui les soutiennent

- préciser la question de l'invitation à participer à des publications scientifiques pour les chercheurs ayant eu une contribution substantielle à la conception des études 
L'ensemble de leurs commentaires et ajouts ont permis d'améliorer le document et de préciser un certain nombre de points.

Dans une dernière étape, la seconde version a été envoyée aux 17 co-chercheurs du programme de recherche pour obtenir, de nouveau, des commentaires additionnels constructifs et proposer une conférence téléphonique pour discuter en détail du contenu. Peu de corrections supplémentaires ont été réclamées et un consensus a donc été trouvé sur la version trois du contenu de la politique. La conférence téléphonique n'a pas été organisée puisque jugée non nécessaire, montrant certainement un accord général sur le document. La version quatre présentée en résumé plus bas (et en détail en annexe) est donc la version définitive adoptée par l'équipe dont nous précisons le contenu dans les prochaines lignes.

\section{Contenu de la politique interne}

Les valeurs qui enchâssent la politique sont la transparence, la prévention, le consensus autour du processus de production, le respect des principes internationaux, l'accès libre aux connaissances produites et la préoccupation pour leur diffusion et utilisation. La politique se présente en plusieurs parties.

Après une introduction générale du contexte global et particulier de son existence, la politique précise son objet, soit « définir des règles et procédures spécifiques au programme de recherche concernant la conservation, l'accès, l'utilisation et la publication des données en s'inscrivant dans les politiques canadiennes et québécoises et celles du pays (i.e. Burkina Faso) où les recherches sont menées ». Le terme "spécifique » est souligné pour bien montrer qu'il ne s'agit pas d'une politique générique, mais qu'elle a été adaptée au contexte particulier du programme de recherches. La notion de données a été précisée dans la politique.

Ensuite, il était essentiel de préciser les statuts des différentes parties prenantes du programme de recherche, car trois types de chercheurs sont impliqués et leurs statuts ont des conséquences importantes, notamment sur les publications scientifiques et le respect des règles de l'International Committee of Medical Journal Editors (ICMJE) [36]. Dans notre contexte, nous avons un chercheur principal du programme et, pour les différentes recherches, des chercheurs principaux, des chercheurs responsables d'études spécifiques et des co-chercheurs collaborant aux différentes études. Dans une perspective de transparence, mais aussi de consensus et de prévention des conflits, une liste précise de l'ensemble des recherches réalisées dans ce programme et des noms des chercheurs associés a été annexés à la politique. Cette liste est révisée au fur et à mesure du déploiement éventuel de nouvelles recherches.

Par la suite, trois autres parties précisent les règles que nous nous sommes données concernant i) la conservation des données, ii) l'accès et l'utilisation des données et enfin iii) la production scientifique et leur paternité (authorship). Sur ce dernier point, nous avons notamment insisté sur l'importance, selon les directives québécoises, de "diffuser les résultats de la recherche de manière responsable et en temps voulu " [3] et sur les règles de l'ICMJE, deux sujets qui ne sont généralement pas toujours bien connus des chercheurs [27].

Les détails du contenu de la politique sont présentés en annexe. Ils sont librement utilisables et doivent nécessairement être adaptés. 


\section{Discussion}

Évidemment, le processus de formulation de cette politique interne, ainsi que les décisions concernant son contenu, n'ont pas été faciles. Nous présentons dans cette discussion quelques un des défis majeurs.

Malgré une demande de soutien, l'université et le centre de recherche d'attache du CP n'ont pas été en mesure de le soutenir dans cette démarche, en se contentant de lui présenter des exemples de politiques d'autres programmes des chercheurs de ces institutions. Le centre de recherche n'a fourni aucun exemple ni soutien et les rares exemples proposés par l'université n'étaient pas adaptés à la RISP et au contexte de la santé mondiale. Cela montre très certainement le caractère encore balbutiant de ces approches dans le monde académique, qui est peut-être encore une " anarchie organisée » [37] comme les analyses des années 1970 le montraient? II nous semble que les universités et centres de recherche devraient être plus réactifs, mieux soutenir leurs chercheurs et anticiper les défis éthiques. Ils pourraient, par exemple, mieux partager leurs expériences et meilleures pratiques en la matière en centralisant et analysant les différentes politiques ou codes de conduite rédigés par chaque équipe ou chercheur isolément, évitant ainsi aux nouvelles équipes de réinventer la roue à chaque fois. Mais en même temps, les chercheurs devraient être plus incités à s'engager dans la production de telles politiques internes à leurs programmes ou projets de recherche, afin d'anticiper le plus tôt possible les difficultés associées aux données, notamment, et assurer une collaboration juste et respectueuse entre tous les chercheurs tout en partageant leurs meilleures pratiques et leçons apprises.

Les chercheurs étaient, au cours des deux premières années du programme, accaparés et concentrés sur la préparation et la mise en œuvre des divers projets de recherches permettant de produire des données. De ce fait, les enjeux autour des données ne sont pas apparus essentiels dans un premier temps. On peut donc certainement regretter que la politique n'ait pas été formulée dès le démarrage du programme, soulevant l'enjeu de sa rétroactivité. Dans d'autres programmes auxquels le CP a participé, il avait alors tenté, en vain, de suggérer aux chercheurs principaux de rédiger une telle politique. Profitant du premier programme d'ampleur dont il a eu la responsabilité, cette politique qui apparaît comme une avancée intéressante, a été formulée. II n'est jamais trop tard pour démarrer une réflexion dans le cadre d'un tel programme, ce que les auteurs du Partnership Assessment Toolkit proposent également [2].

La majorité des chercheurs du programme ont trouvé l'initiative originale et salutaire, quel que soit leur continent de travail. Certains découvraient la plupart de ces enjeux et la possibilité de les aborder sereinement dans le cadre d'un travail d'équipe. Mais tous les chercheurs n'ont pas participé de manière égale à sa formulation, certains effectuant des propositions constructives tandis que d'autres ne participant pas aux discussions. L'analyse de cette différence d'implication reste à faire. Nous pourrions penser que cela montre le besoin impérieux de mieux former les chercheurs en RISP aux enjeux éthiques soulevés par les données [38] et de rester vigilant aux enjeux de pouvoir et d'argent qui souvent peuvent influencer la question de l'utilisation des données dans un contexte de RISP, ce qui devrait nécessairement être discuté au démarrage des collaborations. Selon notre expérience de collaborations scientifiques internationales et de formation des étudiants au Canada et en Afrique, ces défis sont rarement évoqués en public et dans les réunions d'équipe. Cependant, l'expérience de développement de cette politique pourrait certainement s'adapter à d'autres contextes que la RISP, notamment dans le cadre de tout type de partenariat scientifique, ce qui est souvent le cas des collaborations avec les organismes communautaires ou les communautés [39].

L'un des effets inattendus positifs est que l'un des partenaires de recherche au Burkina Faso a décidé de formuler, à son tour, sa propre politique concernant les données de son observatoire populationnel. Alors que ce dernier est en place depuis 2007 [40] et que les enjeux d'utilisation et d'accès aux données lors de collaboration scientifique internationale dans ce cadre sont 
nombreux [38,39], une telle politique n'existait pas. La formulation de notre politique l'a donc incité favorablement à en faire autant à la fin de l'année 2014.

Le suivi de l'application de cette politique reste cependant un défi majeur à relever. II s'agit non seulement des questions liées à l'entreposage et l'accès libre aux données dans des contextes où nos universités ne sont pas encore adaptées à cette nouvelle orientation mondiale. La question du personnel chargé de réaliser ce suivi, encore plus pertinente lorsque les subventions s'arrêtent, se pose avec acuité, mais aussi celle des démarches à entreprendre en cas d'absence de respect du contenu d'une politique décidée collectivement. De surcroit, il serait intéressant, après avoir utilisé et appliqué cette politique pendant quelque temps, de voir comment l'améliorer et tenir compte de notre expérience pour la rendre encore plus utile.

\section{Conclusion}

La formulation d'une telle politique interne concernant les données issues d'un programme de recherches doit certainement être comprise davantage comme un acte de prévention que de sanction. La confiance, la transparence et le respect mutuel doivent finalement être au cœur de tout partenariat scientifique dans le domaine de santé et de la recherche interventionnelle en général [2], mais il vaut certainement mieux prévenir que guérir... Hygea et Panacée [43]!

\section{Références}

1. Droz Y, Mayor A. Partenariats scientifiques avec l'Afrique : Réflexions critiques de Suisse et d'ailleurs. Paris: Karthala; 2009.

2. Murphy J, Hatfield J, Afsana K, Neufeld V. Making a commitment to ethics in global health research partnerships: a practical tool to support ethical practice. Journal of Bioethical Inquiry. 2015;12(1)1-10.

3. Les Fonds de recherche du Québec. Politique sur la conduite responsable en recherche. Québec: Les Fonds de recherche du Québec; 2014.

4. Tenopir C, Allard S, Douglass K, Aydinoglu AU, Wu L, Read E, et al. Data sharing by scientists: practices and perceptions. PLoS ONE. 2011;6(6):e21101.

5. Hawe P, Di Ruggiero E, Cohen E. Frequently asked questions about population health intervention research. Canadian Journal of Public Health. 2012;103(6):e46871.

6. Tremblay M-C, Parent A-A. Reflexivity in PHIR: Let's have a reflexive talk! Canadian Journal of Public Health. 2014;105(3):e221-3.

7. Moore GF, Audrey S, Barker M, Bond L, Bonell C, Hardeman W, et al. Process evaluation of complex interventions: Medical Research Council guidance. BMJ. 2015;350:h1258.

8. Cambon L, Alla F. Recherche interventionnelle en santé publique, transfert de connaissances et collaboration entre acteurs, décideurs et chercheurs. Le défi français de l'innovation. Questions de Santé Publique. 2014;27:1-4.

9. Hawe P. Minimal, negligible and negligent interventions. Social Science and Medicine. 2015;138:265-8.

10. Jabot F, Ridde V, Wone I, Fond-Harmant L. L'évaluation, une voie pour faire progresser la promotion de la santé en Afrique ? Santé Publique. 2014;26(1 Suppl):21-34.

11. Ridde V, Olivier de Sardan, Jean-Pierre. A mixed methods contribution to the study of health public policies: complementarities and difficulties. BMC Health Services Research. 2015;15(Suppl 3): S7.

12. Gouvernement du Canada, IRSC. Activités des groupes de travail de l'IRISPC : compétences en recherche interventionnelle en santé des populations.

13. Pickersgill M. The co-production of science, ethics, and emotion. science, technology \& human values. 2012;37(6):579-603.

14. Mohindra KS, Ridde V. Canada's role in global health: Guiding principles for a growing research agenda. Canadian Foreign Policy Journal. 2009;15(3):106-15. 
15. Suárez-Herrera JC, Blain M-J. La recherche en santé mondiale: perspectives socioanthropologiques. Association francophone pour le savoir, Acfas; 2012. 276 p.

16. Chetwood JD, Ladep NG, Taylor-Robinson SD. Research partnerships between high and lowincome countries: are international partnerships always a good thing? BMC Medical Ethics. 2015;16(1):36.

17. Kass NE. An ethics framework for public health. American Journal of Public Health. 2001;91(11):1776-82.

18. Dagenais C, Malo M, Robert E, Ouimet M, Berthelette B, Ridde V. Knowledge transfer on complex social interventions in public health: a scoping study. PLoS One. 2013;8(12):e80233.

19. Sonnenwald D. Scientific collaboration. Annual Review of Information Science and Technology. 2008;41(1):643-81.

20. Ridde V, Capelle F. La recherche en santé mondiale et les défis des partenariats Nord-Sud. Revue Canadienne de Santé Publique. 2011;102(2):152-6.

21. Ouattara $F$, Ridde V. Expériences connues, vécues ... mais rarement écrites. À propos des relations de partenariat Nord-Sud. Nouvelles Pratiques Sociales. 2013;25(2):231-46.

22. Olivier de Sardan J-P. Promouvoir la recherche face à la consultance. Autour de l'expérience du Lasdel (Niger-Bénin). Cahiers d'études Africaines. 2011;202-203(2-3):511-28.

23. Sombié I, Aidam J, Konaté B, Somé TD, Kambou SS. The state of the research for health environment in the ministries of health of the Economic Community of the West African States (ECOWAS). Health Research Policy and Systems. 2013;11(1):35.

24. Godard B, Hunt M, Moube Z. Éthique de la recherche en santé mondiale : la relation NordSud, quel partenariat pour quelle justice sociale? Global Health Promotion. 2014;21(2):80-7.

25. Ravinetto R, Buvé A, Halidou T, Lutumba P, Talisuna A, Juffrie M, et al. Double ethical review of North-South collaborative clinical research: hidden paternalism or real partnership? Tropical Medicine and International Health. 2011;16(4):527-30.

26. Nyika A, Kilama W, Chilengi R, Tangwa G, Tindana P, Ndebele P, Ikingura J. Composition, training needs and independence of ethics review committees across Africa: are the gatekeepers rising to the emerging challenges? Journal of Medical Ethics. 2009;35(3):189-93.

27. Smith E, Hunt M, Master Z. Authorship ethics in global health research partnerships between researchers from low or middle income countries and high income countries. BMC Medical Ethics. 2014;15:42.

28. Uthman OA, Wiysonge CS, Ota MO, Nicol M, Hussey GD, Ndumbe PM, Mayosi BM. Increasing the value of health research in the WHO African Region beyond 2015-reflecting on the past, celebrating the present and building the future: a bibliometric analysis. BMJ Open. 2015;5(3):e006340.

29. Ridde V, Belaid L, Samb OM, Faye A. Les modalités de collecte du financement de la santé au Burkina Faso de 1980 à 2012. Santé Publique. 2014;26(5):715-25.

30. Ware M, Mabe M. The STM Report. An overview of scientific and scholarly journal publishing. Celebrating the 350th anniversary of journal publishing. The Hague: STM: International Association of Scientific, Technical and Medical Publishers; 2015 mars p. 180.

31. Lariviere V, Sugimoto C, Tsou A, Gingras Y. Team size matters: Collaboration and scientific impact since 1900. Journal of the Association for Information Science and Technology. 2014; arXiv:1410.8544.

32. Upshur RE, Lavery JV, Tindana PO. Taking tissue seriously means taking communities seriously. BMC Medical Ethics. 2007;8:11.

33. Ridde V, Mohindra K, LaBossière F. Driving the global public health research agenda forward by promoting the participation of students and new researchers: Perspectives from Quebec. Canadian Journal of Public Health. 2008;99(6):460-5.

34. Ana J, Koehlmoos T, Smith R, Yan LL. Research misconduct in low- and middle-income countries. PLoS Med. 2013;10(3):e1001315.

35. Fang FC, Steen RG, Casadevall A. Misconduct accounts for the majority of retracted scientific publications. PNAS. 2012;109(42):17028-33. 
36. International Committee of Medical Journal Editors. Recommendations for the Conduct, Reporting, Editing, and Publication of Scholarly Work in Medical Journals. ICMJE; 2014 p. 17.

37. Cohen MD, March JG, Olsen JP. A garbage can model of organizational choice. Administrative Science Quarterly. 1972;17(1):1-25.

38. Government of Canada, CIHR. PHIRIC working group activities: population health intervention research competencies.

39. Bilodeau A, Galarneau M, Fournier M, Potvin L. L'Outil diagnostique de l'action en partenariat: fondements, élaboration et validation. Canadian Journal of Public Health. 2011;102(4):298302.

40. Kouanda S, Bado A, Yameogo M, Nitiema J, Yameogo G, Bocoum F, Millogo T, Ridde V, Haddad S, Sondo B. The Kaya HDSS, Burkina Faso: a platform for epidemiological studies and health programme evaluation. International Journal of Epidemiology. 2013;42(3):741-9.

41. Carrel M, Rennie S. Demographic and health surveillance: longitudinal ethical considerations. Bulletin of the World Health Organization. 2008;86(8):612-6.

42. Mondain N, Bologo $\mathrm{E}$. La restitution des résultats dans les suivis démographiques en Afrique subsaharienne : au-delà de la norme éthique, un souci pédagogique. Interrogations. 2011;13.

43. Renaud M. L'avenir : Hygié ou Panacée ? In: Evans RG, Stoddart GL, éditeurs. Être ou ne pas être en bonne santé : biologie et déterminants sociaux de la maladie. Montréal: Presses de l'Université de Montréal; 1996. p. 317-32. 


\section{Annexe A}

\section{POLITIQUE INTERNE CONCERNANT LA CONSERVATION, L'ACCÈS, L'UTILISATION ET LA PUBLICATION DES DONNÉES}

\section{Introduction}

Le programme de recherches concerne essentiellement des interventions à base communautaire concernant les problèmes de santé et d'équité en santé les plus importants dans le pays, ainsi que ceux qui sont les plus négligés. Le but du programme est d'étudier l'efficacité et les processus des interventions prometteuses pour améliorer l'équité en santé au Burkina Faso. Le chercheur principal du programme est Valéry Ridde et le programme a été financé par les IRSC pour la période 20112016.

Dans le cadre de ce programme, de nombreuses recherches ont été réalisées (voir annexe 1) et plusieurs autres seront entreprises d'ici la fin de la période de financement. De nombreuses données quantitatives et qualitatives ont donc été collectées. Puisque de nombreux chercheurs sont impliqués dans ce programme ainsi que des étudiants et professionnels de recherche (assistants, ingénieurs, etc.), il est essentiel qu'une politique claire, juste et transparente de gestion et d'utilisation des données soit définie.

Cette politique interne au programme de recherche n'entend pas se substituer aux règles et bonnes pratiques existantes (annexe 4) dont les chercheurs doivent nécessairement connaître le contenu. En effet, il s'agit de respecter les règles d'éthique et de conduite responsable de la recherche en vigueur, notamment :

- La Politique des trois organismes sur le libre accès aux publications, 2015

- La Politique sur la conduite responsable en recherche des FRQ, 2014

- La 2ème édition de l'énoncé de politique des trois Conseils canadiens : éthique de la recherche avec des êtres humains, 2012

- Le cadre de référence des trois organismes sur la conduite responsable de la recherche, 2011

- Le règlement en matière de probité intellectuelle en recherche et développement. CHUM, 2005

\section{Objet}

Ce document a pour objet de définir des règles et procédures spécifiques au programme de recherche concernant la conservation, l'accès, l'utilisation et la publication des données en s'inscrivant dans ces politiques canadiennes et québécoises et celles du pays où les recherches sont menées.

Dans cette politique, la notion de données est définie comme toute information recueillie ou utilisée dans le cadre des activités de recherche du programme destinée à être colligée ou qui est colligée dans des banques constituées. Cette information inclut les réponses aux questionnaires quantitatifs auprès des personnes ainsi que les résultats des tests réalisés ou le matériel biologique collecté, les coordonnées géographiques, les informations provenant des registres administratifs, le contenu des entrevues qualitatives formelles. Bien que les analyses documentaires, les prises de notes ou encore les journaux de bord soient considérés comme des données utiles aux recherches qualitatives, elles ne sont pas considérées comme telles dans cette politique.

\section{Typologie des chercheurs}

Cette politique interne concerne le programme de recherche et tous les projets dont les protocoles ont été soumis aux comités d'éthique des deux pays. En plus du chercheur principal du programme, chacun de ces protocoles spécifie la présence de trois types de chercheurs : chercheurs principaux, chercheurs responsables d'études spécifiques et co-chercheurs collaborant aux différentes études. Les noms de ces chercheurs sont précisés en annexe 1. Cette liste sera mise à jour en fonction des 
nouveaux projets de recherche éventuellement soumis d'ici la fin de la période de financement. Les chercheurs pourront faire signer un engagement au respect de cette politique de la part de leurs collaborateurs au moyen de l'annexe 5.

\section{Conservation des données}

1. Toutes les données provenant des études ${ }^{1}$ (annexe 1) doivent être systématiquement envoyées par le chercheur responsable de chaque étude à la coordination du programme de recherche, une fois la collecte des données terminées.

2. Toutes les données originales seront conservées au niveau de la coordination du programme qui en assure la conservation sur le serveur (CRCHUM ou université de Montréal) ou sur papier, le tout sécurisé.

3. Les chercheurs responsables des études gardent une copie des données originales pour les analyses subséquentes.

4. Toutes les données doivent être dénominalisées, sécurisées et l'accès protégé.

5. Toutes les données doivent être conservées pour une durée minimale de 5 années après la fin de la période de validité de la subvention.

6. Aucune donnée ne peut être détruite sans l'accord du chercheur principal du programme de recherche et le chercheur principal ne pourra détruire aucune donnée sans l'accord des chercheurs responsables des études.

7. Le programme de nettoyage des données quantitatives doit être transmis une fois le travail réalisé.

\section{Accès et utilisation des données}

8. Aucune donnée ne peut être transmise à des personnes (étudiants, statisticiens, traducteurs, assistants pour nettoyage ou transcription des données, chercheurs, etc.) qui ne sont pas identifiées dans les protocoles de recherche remis aux comités d'éthique (annexe 1).

9. La transmission des données à d'autres personnes pour des fins d'analyses est possible mais uniquement si l'analyse est réalisée sous la responsable d'un chercheur identifié dans un des protocoles (annexe 1).

10. Un chercheur de l'équipe (annexe 1) qui souhaite engager une personne et lui permettre d'accéder à ces données doit lui demander de signer une demande par écrit (annexe 2). Ce formulaire doit être envoyé à chacun des chercheurs responsables de chaque étude (annexe 1 ), ou en l'absence, au(x) chercheur(s) principal(aux), suivant le modèle en annexe 2 . Les chercheurs qui s'engagent à superviser ce travail d'analyse doivent contre-signer la demande d'accès aux données par une tierce personne pour l'accepter. Ce document doit par la suite être envoyé pour information à la coordination du programme.

11. La transmission des données doit garantir l'anonymat et la vie privée des participants à la recherche.

12. Les personnes non identifiées dans les protocoles de recherche remis aux comités d'éthique (annexe 1) et qui auront par la suite eu l'autorisation d'accéder aux données s'engageront à respecter la présente politique.

13. Une réflexion sera entreprise dans les prochains mois concernant la mise en accès libre des données du programme de recherche à des chercheurs externes car pour le moment, la

\footnotetext{
${ }^{1}$ Les données longitudinales collectées par l'observatoire populationnel de l'IRSS (dit Kadess), y compris celles financées durant une année par le programme Équité, ne sont pas concernées par cette politique mais par son code d'utilisation du 22 décembre 2014.
} 
transmission puis l'analyse des données n'est possible que sous la responsabilité de chaque chercheur responsable d'étude ou chercheur principal.

14. Dans le cas où ces règles internes concernant la conservation, l'accès et l'utilisation des données de la présente politique ne sont pas présentes dans les protocoles soumis aux différents comités d'éthiques, une demande d'amendement sera réalisée par les chercheurs principaux.

\section{Productions scientifiques et paternité (authorship)}

15. Les chercheurs qui dirigent des études ou participent à des projets du programme de recherche sont encouragés fortement à publier les résultats découlant de leur recherche afin que la communauté scientifique et la population en général puissent en bénéficier. Le $F R Q$ estime qu'une conduite responsable implique de "diffuser les résultats de la recherche de manière responsable et en temps voulu ».

16. Les règles concernant les auteurs de productions scientifiques dans le cadre du programme de recherche sont définies comme suit et suivent les propositions du International Committee of Medical Journal Editors (2013).

17. Le processus intellectuel qui conduit à une production scientifique (article, affiche, communication orale, chapitre de livre, notes de politique, etc.) inclut les étapes suivantes :

i. Contribution substantielle à la conception OU au devis de l'étude, OU l'acquisition et l'analyse des données, OU l'interprétation des données; ET V

ii. Écriture d'une première version de la publication OU relire et réviser en apportant une contribution critique intellectuelle; ET V

iii. Approuver la version finale pour publication; ET V

iv. Accepter d'être garant de tous les aspects du travail, notamment à l'égard de la précision OU de l'intégrité du contenu de l'article ET de la pertinence de l'étude et des analyses.

18. Toutes les personnes qui sont désignées comme auteurs dans une production scientifique doivent respecter ces quatre critères et ceux qui respectent ces quatre critères doivent être auteurs. Ceux qui ne respectent pas ces critères, mais qui ont contribué à l'étude doivent être mentionnés dans la section des remerciements de la production.

19. Ces critères ne peuvent pas dénier le droit à certains d'être auteur d'une production scientifique, en cherchant à ne pas les impliquer ou ne pas leur donner l'opportunité d'être associé aux étapes 2 (ii) et 3 (iii). Ainsi, toutes personnes qui participent à la première (i) étape, et rencontrent donc le premier critère, doivent nécessairement avoir l'opportunité d'être auteur des productions scientifiques qui en découlent, notamment en participant à l'écriture, la révision et l'approbation finale. Dans le contexte du programme de recherche, cela signifie que tous les chercheurs principaux et les chercheurs responsables des différentes études (annexe 1) doivent avoir l'opportunité de participer aux productions scientifiques. Si d'autres chercheurs que ces deux catégories ont participé à la première étape, ils doivent aussi disposer de cette opportunité. L'absence de cette opportunité est considérée comme un manquement aux bonnes pratiques dans le domaine de la santé.

20. Pour que ce processus soit organisé en toute transparence, chaque personne décidant de réaliser une production scientifique doit donc en informer toutes les personnes qu'elle souhaite impliquer. Cette proposition doit être effectuée au moyen du plan proposé en annexe 3 (avantprojet d'article). Toutes les personnes contactées ont 10 jours ouvrables pour répondre à cette opportunité. L'absence de réponse dans ce délai est comprise comme un refus de participer au processus de réalisation de cette production scientifique. Toutes les propositions d'articles, une fois les accords entre auteurs trouvés, doivent être envoyées pour information à la coordination du programme. Cet accord préalable ne garantit pas nécessairement la présence 
comme auteur d'un article, car ce statut passe par le respect des quatre critères (point 17), faisant en sorte que cet accord est révisable en tout temps dans le respect de ces règles.

21. L'ordre des auteurs doit être décidé de manière transparente entre tous les coauteurs et respecter la contribution réelle tout au long du processus d'écriture. Le premier auteur est celui qui prend le leadership de l'écriture de l'article, il y contribue le plus et il est responsable du contenu scientifique (notamment en cas de plagiat, erreur, etc.) et du contact avec les coauteurs. On ne peut pas indiquer le nom d'un auteur s'il n'a pas donné son accord sur le document.

22. Un effort particulier doit être effectué par les chercheurs pour que toutes les productions scientifiques soient en accès libre. Conformément à la politique des IRSC, pour les articles scientifiques, les chercheurs doivent s'organiser pour les publier dans des revues en accès libre (Open Access) immédiat ou dans les 12 mois qui suivent la publication. Le programme de recherche pourra payer, dans les limites de son budget, les frais de publications (Article Processing Charges: $A P C$ ). Si les chercheurs ne souhaitent pas publier dans une telle revue, ils devront s'assurer que la revue choisie permette un dépôt central ou institutionnel accessible dans les 12 mois suivant la publication.

23. Certaines revues réclament que les bases de données et les instruments de collecte de données à l'origine des publications soient accessibles sur Internet. Dans ce cas, l'auteur principal de l'article devra communiquer avec la coordination du programme de recherche afin de trouver la solution technique appropriée avant toute publication.

24. Une copie de toutes les productions scientifiques (article, affiche, communication orale, chapitre de livre, note de politique, utilisation dans des cours, etc.) émanant des données des projets de recherche doit être systématiquement envoyée pour information à la coordination du programme de recherche une fois sa présentation réalisée. Toutes les productions scientifiques seront partagées sur le site du programme de recherche (www.equitesante.org) après en avoir obtenu l'accord par le chercheur responsable de cette production scientifique. Dans un souci de partage des connaissances, les chercheurs doivent s'engager à ce que la majorité de ces productions soient partagées sur le site, mais il est possible que cela ne soit pas toujours possible ou souhaitable (par exemple dans le cas d'analyses préliminaires de données).

25. Toutes les productions scientifiques doivent mentionner le soutien financier des IRSC par la phrase suivante : "The research project is part of the "Community research studies and interventions for health equity in Burkina Faso". We thank the Canadian Institutes of Health Research (CIHR), who funded the program (Grant number ROHU115213) ".

26. Tous les conflits d'intérêts de chaque auteur associé à la production scientifique doivent être énumérés.

\section{Manquement aux règles de la politique}

27. Toute personne ayant connaissance d'un manquement aux règles de cette politique doit en informer immédiatement le chercheur principal du programme.

28. Dans le cas où un manquement aux règles est constaté, le chercheur principal du programme cherchera à le comprendre et le résoudre à l'amiable avec les personnes concernées et éventuellement le comité des co-chercheurs du programme.

29. Dans ce cas où cet accord à l'amiable n'est pas possible, le chercheur principal informera les autorités universitaires du manquement constaté. 


\section{Annexe 1 : Protocoles de recherche et typologie des chercheurs en date du 01/2015}

Chacun des titres en gras et surlignés en gris a fait l'objet d'un protocole de recherche déposé aux comités d'éthiques, sauf le volet concernant les développements méthodologiques.

SANTE INFANTILE 2012/2013

- Chercheur principal

- Valéry Ridde

- Chercheurs responsables d'études

- Slim Haddad et Valéry Ridde

- Enquête ménages visant à évaluer l'intervention d'exemption de paiement des soins pour les enfants de moins de cinq ans et l'intervention communautaire de lutte contre le paludisme

- Utilisation des données de routine pour comprendre l'hétérogénéité de la performance des soins de santé primaires

- Perception de la qualité des soins et analyse des coûts dans un contexte de gratuité

- Seni Kouanda

- Autopsies verbales de décès par le système de surveillance démographique

- Valéry Ridde et Slim Haddad

- Analyse du processus des interventions de lutte contre le paludisme impliquant des agents de santé communautaire

- Co-chercheurs

- Emmanuel Bonnet, Aude Nikiema, Seni Kouanda

\section{NOUVELLES PROBLEMATIQUES, AMENDEMENTS DU PROTOCOLE SANTE INFANTILE} 2012/2013

- Chercheur principal

- Valéry Ridde

- Chercheur responsable d'étude :

- François Chiocchio

- Effet du leadership sur la collaboration dans les CSPS de Kaya

- Co-chercheurs :

- Seni Kouanda, Slim Haddad

- Chercheur principal

- Valéry Ridde

- Épidémie de dengue à Ouagadougou en 2013

- Co-chercheurs :

○ Seni Kouanda, Florence Fournet, Roch Dabiré, Emmanuel Bonnet

ASSURANCE ET INDIGENCE 2012/2013

- Chercheur principal

○ Valéry Ridde

- Analyse du programme national de solidarité du Réseaux d'Appui aux Mutuelles de Santé

- Co-chercheur :

- Seni Kouanda 
- Chercheur principal

- Valéry Ridde

- Compréhension et adhésion aux concepts sous-jacents à la couverture santé nationale

APPLICATION DES CONNAISSANCES 2012-2013

- Chercheur principal

- Valéry Ridde

- Chercheurs responsables d'études :

- Christian Dagenais

- Évaluation de la première année de courtage de connaissance

AUDITS CLINIQUES COMBINÉS À LA FORMATION DANS LA RÉDUCTION DU TAUX DE CÉSARIENNES ABUSIVES DANS LES HÔPITAUX DU BURKINA FASO 2013/2015

- Chercheur principal

- Alexandre Dumont

- Co-chercheurs

- Seni Kouanda, Valéry Ridde

TRAUMATISMES DE LA CIRCULATION ROUTIERE A OUAGADOUGOU 2013/2015

- Chercheurs principaux

- Emmanuel Bonnet, Valéry Ridde

- Co-chercheurs

- Kabré Théophile, Ky Barbara, Lompo Kévin, Ouedraogo Fabrice, Hamadou Kaboré, Joanny Bassolé, Aude Nikiema

FINANCEMENT BASÉ SUR LES RESULTATS ET PROTECTION SOCIALE 2013/2015

- Chercheurs principaux

- Valéry Ridde, Aurélia Souares, Manuela De Allegri

- Chercheurs responsables d'études :

- François Chiocchio et Nathalie Houlfort

- Motivation et performance des CSPS

- Matthew Hunt

- Co-chercheurs

- Analyse éthique des interventions

- Hervé Hien, Maurice Yaogo, Zongo Sylvie, Paul André Somé, Nathalie Houlfort, François Chiocchio, Emmanuel Bonnet, Matthew Hunt

INTERVENTION COMMUNAUTAIRE DE LUTTE CONTRE LES MALADIES FEBRILES ET SURVEILLANCE PASSIVE A OUAGADOUGOU 2013/2015

- Chercheur principal

○ Valéry Ridde

- Co-chercheurs

- Yaro Seydou, Roch Dabire, Florence Fournet, Paul-André Somé, Emmanuel Bonnet

GOUVERNANCE ET RECHERCHE ACTION NUMERO VERT A OUAGADOUGOU 2013/2015

- Chercheur principal

- Valéry Ridde

- Co-chercheurs

- Emmanuel Bonnet, Christian Dagenais 
DEVELOPPEMENTS METHODOLOGIQUES 2013/2015

- Chercheurs responsables d'études

○ Emmanuel Bonnet et Valéry Ridde

- Analyses spatiales

- Valéry Ridde et Manuella De Allegri

- Méthodes mixtes

- Valéry Ridde

- Recherches actions

APPLICATION DES CONNAISSANCES 2013/2015

- Chercheur principal

- Valéry Ridde

- Chercheurs responsables d'études :

- Christian Dagenais

- Évaluation du courtage de connaissances et de l'atelier de partage des résultats

- Co-chercheurs :

- Paul André Somé 


\section{Annexe 2 : Contrat d'accès et d'utilisation des données}

Je, soussigné (e), souhaiterais analyser des données générées dans le cadre du projet « XXX » dont le(s) chercheur(s) principal(aux) est XXXX.

Dans le tableau ci-dessous, sont indiqués le titre et l'objectif de mon travail :

\begin{tabular}{|l|l|}
\hline Titre du travail & \\
\hline Objectifs & \\
\hline Activités prévues & \\
\hline
\end{tabular}

Plus particulièrement, je souhaiterais obtenir l'accès aux données suivantes :

- XXX (préciser les données de manière spécifique, la base de données, ou les données tirées de quelle méthode en particulier)

- $\quad X X X X$

- $\quad X X X X$

En signant ce document :

$>$ Je m'engage à n'utiliser les données que dans le cadre du projet mentionné ci-dessus sous la responsabilité du chercheur XXX. Toute utilisation des données dans un autre objectif n'est pas autorisée.

$>$ Je m'engage à ne pas transmettre les données.

$>$ Je m'engage à protéger l'accès aux données et leur confidentialité.

$>$ Je m'engage à informer le chercheur principal du projet de tout problème rencontré au cours de l'analyse des données.

$>$ Je m'engage à produire des résultats, publier les analyses et les données en mentionnant la source des données.

$>$ Je m'engage à respecter les règles mentionnées dans la politique interne du programme de recherche concernant les auteurs.

$>$ Je suis informé que tous les articles produits ainsi que la liste des auteurs de ces articles devront être discutés en avance.

$>$ Je suis informé que le processus intellectuel qui conduit à la publication d'un article inclut les étapes précisées par la politique interne.

$>$ Je m'engage à envoyer le programme des analyses de données quantitatives au chercheur principal du programme de recherche.

$>$ Je m'engage à envoyer une version complète de toutes les productions scientifiques et tous les articles publiés au chercheur principal du programme de recherche. 


\section{Annexe 3 : Modèle d'avant projet d'article}

L'avant-projet est proposé pour amorcer un projet d'article et servir de fondement pour les discussions entre les auteurs potentiels. II poursuit quatre buts. Le premier est de décrire le projet d'article. Le second est de signifier un point de départ pour procéder aux travaux menant à la publication. Le troisième est d'identifier et d'éliminer, le cas échéant, les obstacles à la publication. Le quatrième est de s'assurer de l'implication tôt dans le processus de l'ensemble des coauteurs potentiels et de leur souhait d'être associé pour en partager la paternité (authorship) suivant la politique interne du programme de recherche.

Le plan schématique de l'avant projet est une description sommaire du contenu anticipé de l'article, il devrait ne pas dépasser 500 mots. II est évident qu'il est difficile de décrire l'article de façon précise et étendue étant donné que le travail n'est pas amorcé. Toutefois, on y distingue les idées maîtresses.

\section{Plan schématique de l'article}

\begin{tabular}{|l|l|}
\hline Auteurs proposés : & $\begin{array}{l}\text { nom, prénom et ordre provisoire des auteurs (l'ordre pourra évoluer en } \\
\text { fonction du processus d'écriture) }\end{array}$ \\
\hline Titre proposé : & selon la revue, court, concis et factuel le plus souvent \\
\hline Revues visées : & deux ou trois noms de revues dans l'ordre proposé de soumission \\
\hline Accès libre : & préciser la politique de la revue en la matière, les coûts d'APC \\
\hline Introduction : & justifier la pertinence contextuelle et scientifique de l'article \\
\hline Méthodes : & $\begin{array}{l}\text { présenter succinctement les données, les méthodes et les analyses } \\
\text { proposées }\end{array}$ \\
\hline Résultats attendus : & $\begin{array}{l}\text { présenter les tableaux ou les thèmes d'analyses attendus, le } \\
\text { programme de recherche concernant l'équité, une attention particulière } \\
\text { à ce sujet doit être apportée }\end{array}$ \\
\hline Utilité des résultats : & $\begin{array}{l}\text { au-delà de la contribution à l'avancement des connaissances, en quoi } \\
\text { les résultats seront utiles pour la santé publique, les systèmes de santé } \\
\text { et notamment les enjeux concernant l'équité }\end{array}$ \\
\hline Références essentielles : & fournir une liste courte $(n=5)$ des références essentielles sur le sujet \\
\hline
\end{tabular}




\section{Annexe 4 : documents consultés pour la rédaction de cette politique}

- La Politique sur la conduite responsable en recherche des FRQ, 2014

- La 2ème édition de l'énoncé de politique des trois Conseils canadiens : éthique de la recherche avec des êtres humains, 2012

- Le cadre de référence des trois organismes sur la conduite responsable de la recherche, 2011

- Le règlement en matière de probité intellectuelle en recherche et développement. CHUM, 2005

- Déontologie de la signature des publications et communications. Règles appliquées dans le cadre des partenariats associant notamment l'IRD (UMI 233), le CRCF, et leurs institutions partenaires, 2014

- Mémorandum d'entente. Dissémination du rapport de l'enquête de base Accès aux données manquantes. Évaluation d'impact et le rapport de l'enquête de base « Financement basé sur les résultats » Burkina Faso, 2014

- Politique d'accès et d'utilisation des Données et des Échantillons. CARTaGENE, 2013

- Agreement and procedures for use of the Ouagadougou Health and Demographic Surveillance System (Ouaga HDSS) data, June 2013

- Cadre de gestion d'une banque de données et de matériel : Références et proposition, Version du 30 juillet 2012, CERES

- Recommandations pour la conduite, la présentation, la rédaction et la publication des travaux de recherche soumis à des revues médicales. Décembre 2014

- Document de gestion des banques de données constituées ou utilisées pour des fins de recherche. 20 janvier 2006

- Guide d'élaboration des cadres de gestion des banques de données et de matériel biologique constituées à des fins de recherche. Unité de l'Éthique Octobre 2012. Ministère de la santé et des services sociaux

- $\quad$ Guidelines for Best Practices in Data Management - Roles and Responsibilities. March 2012. Data Architecture Advisory Committee

- Charte nationale de déontologie des métiers de la recherche, France, 29 janvier 2015 


\section{Annexe 5 : Formulaire d'engagement}

\section{FORMULAIRE D’ENGAGEMENT CONCERNANT LES DONNÉES DU PROGRAMME EQUITE}

La présente annexe doit être signée par toute personne impliquée dans la collecte, la manipulation ou l'analyse des données issues du programme de recherche Équité et qui n'aurait pas signée d'engagement équivalent au sein de son contrat de travail ou de son entente de service professionnel.

Le présent formulaire devra être expliqué et contresigné par le responsable de la recherche au sein de laquelle se déroule l'étude concernée ou par la personne en charge de son recrutement.

Par le présent formulaire, je soussigné

1. POLITIQUE

Je m'engage à respecter la politique concernant les données du programme de recherche Équité.

Je reconnais avoir lu, approuvé et m'engage à respecter la politique d'utilisation des données du programme Équité.

2. CONFIDENTIALITÉ

L'expression « information confidentielle » concerne les informations et documents, dont tous les renseignements verbaux ou écrits ainsi que les supports d'information, notamment : tous les renseignements cliniques, dossiers de patients, registres, coordonnées géographiques, cahiers des charges, renseignements techniques, logiciels, dessins, spécimens, matériels, projets, prototypes, dispositifs, appareils, secrets de fabrique, secrets commerciaux, etc. Cela inclut également les mots de passe et codes d'accès obtenus dans le cadre de collaborations ou fonctions exercées dans le cadre de cette recherche.

Par la présente, je m'engage à ne divulguer aucune information confidentielle, telle que ciavant définie, obtenue dans le cadre de cette collaboration avec le programme Équité, sauf si le chercheur-responsable m'en donne l'autorisation ou le mandat spécifique par écrit. Je m'engage également à préserver la confidentialité de l'information, nommément :

(i) de ne pas faire de copie ou de transcription de l'information confidentielle;

(ii) de ne pas faire circuler ou diffuser, de quelque manière que ce soit, l'information confidentielle;

(iii) de ne pas discuter de l'information confidentielle avec toute personne non autorisée n'ayant pas souscrit à un engagement de confidentialité dont la teneur et les conditions ne devront pas être moindres que celles stipulées dans ce document;

(iv) de ne pas utiliser l'information confidentielle à mes propres fins, ni à des fins autres que celles spécifiquement prévues ;

(v) de prendre avec diligence toutes les mesures raisonnables afin de préserver la confidentialité de l'information confidentielle;

(vi) de respecter toute loi, directive, politique ou norme applicable aux données personnelles ou nominatives auxquelles je peux avoir accès à l'occasion de cette collaboration.

(vii) de prendre les moyens appropriés pour empêcher le traitement non autorisé ou illégal, la divulgation injustifiée de même que la perte accidentelle, la destruction ou la corruption de ces données ou de quelque partie de celles-ci.

(viii) à la fin de la collaboration, je vais détruire tous les documents de projets sous toutes les formes d'une manière confidentielle, c'est-à-dire par déchiquetage des documents 
papier et supprimer entièrement toutes les copies électroniques de mon disque dur d'ordinateur et d'autres médias, y compris les sauvegardes.

Mes obligations découlant du présent engagement sont pour une durée indéterminée.

3. MATERIEL

Je m'engage à protéger en tout temps contre la perte involontaire ou malveillante, les dommages, les modifications ou la divulgation, tout bien matériel et immatériel appartenant au programme Équité (par exemple : les équipements, matériel de collecte, cahiers de collecte, équipements informatique et logiciels, brevets, accords de licence, ententes de transferts de matériel)

Lu et accepté à le $201 X$

Le collaborateur :

Le chercheur responsable : 


\section{A Policy Regarding Data Generated by a Global Health Intervention Research Program}

\section{Introduction}

At a time when public health intervention research is in full expansion, its conduct in a global health context poses major ethical challenges with respect to data and their publication, particularly in Africa, where issues of power and money are inherent to international collaboration [1,2]. To address these challenges, our team developed an internal policy on data storage, access, use, and publication. In this article, we present this policy development process with a view to sharing our experience and stimulating an a priori debate that is essential for the responsible conduct of research [3,4]. Our focus will be not so much on the usual ethical issues of protecting individuals or research participants, but more on the challenges of international collaboration and on researchers' ethical commitments and practices. Further, our reflection comes under what today is (re)named public health intervention research, as the experience we describe is part of a program to analyze interventions implemented in Burkina Faso; this program is presented in greater detail below.

In this article, we first present the context in which global health intervention research is conducted and then explore the question of what constitutes responsible conduct of research. We then describe the specific context of our research program, which led to our creating an internal policy regarding data, and we conclude with an analysis of the policy formulation process and its challenges.

\section{Challenges of conducting global health intervention research}

While public health intervention research (PHIR) is not new, recent years have seen a revival in which the field, as well as related disciplines and research competencies, have become more clearly defined $[5,6]$. Without revisiting its history, here we define PHIR as the application of a scientific approach to the analysis of population health interventions. The development of PHIR has assumed a relatively broad scope in Canada, particularly due to the impetus provided by the Canadian Institutes of Health Research (CIHR) and its Institute of Population and Public Health, along the lines of what is being done, for example, in Europe [7,8] and Australia [9]. However, this movement appears still to be somewhat hesitant in low- and middle-income countries, and particularly in Africa, where PHIR is rarely undertaken [10]. Epidemiological studies still prevail on the African continent, and analyses of complex interventions by means of an interdisciplinary approach and mixed methods are still exceptional [11], even though they are an integral component of PHIR.

Among the key competencies that researchers should acquire, the working group for the Population Health Intervention Research Initiative for Canada (PHIRIC) has proposed applying "a relevant set of ethical principles to the research process" [12]. In particular, it specifies that researchers need to adopt a reflective stance with regard to not only their behaviours, but also the impacts of their actions. As such, some authors consider that "science" and "ethics" are inseparable, have a mutual influence, and lead researchers to adopt progressive normative positions [13]. However, the group did not go much further and did not define these principles specifically, thereby giving different teams the latitude to develop them for their own particular situations, which is in fact the subject of our article.

Ethical questions surrounding the development of global health PHIR must be taken seriously [14-16]. This particular issue, which is the focus of this article, has less to do with traditional bioethical discussions (autonomy, justice, beneficence, doing no harm, protection of persons) than with the conduct of research and particularly with the behaviours of researchers, who are obviously influenced 
by their institutions. We also will not dwell on the ethical issues associated with interventions [17] as such, since in PHIR, unlike action-research, researchers often occupy a more distant position and rarely intervene in the content or implementation of actions. Thus, given the complexity inherent in the object of the PHIR, researchers must also be able to work in teams and to collaborate with colleagues from other disciplines and other organizational cultures, as well as with stakeholders and decisionmakers who want the results of studies to be both useful and usable [18]. In this context, the challenges become crystallized around three main types of scientific collaboration associated with PHIR: disciplinary, geographic, and organizational [19]. These different issues, in turn, pose other ethical challenges, which we touch upon later, having to do particularly with interdisciplinary collaboration, team work, and knowledge application. These appear to be relatively more intense in the area of global health research.

\section{Responsible conduct of research in the international collaboration context}

Our previous works, and those of many others, have shown the extent to which questions of power and money can influence team-based research processes, notably in a context of North-South relations, colonial history, and financial dependency [20-22]. For example, in Burkina Faso, 87\% of health research projects are funded by external sources [23]. Organizational and individual cultures in the field of PHIR can differ greatly from one country, or even one continent, to another. Scientific partnerships, which are inherent to this form of public health research practice, are complex to mobilize [1] and present numerous ethical issues [24]. These have to do with, among other things, the definition of research questions and how they are aligned with the country's priorities, the distribution and management of financial resources, the decision-making processes, ownership of the collected data, etc. We might also point to the dual burden, and its implications, imposed by this type of scientific collaboration that necessarily involves both Northern and Southern research ethics committees [25]. Moreover, members of the research ethics committees analyzing these protocols often are not suitably trained to assess PHIR projects, are not very available, and do not have all the means required to properly carry out their roles [26].

In the areas of scientific publication and attribution of authorship, the ethical challenges posed by international collaborations are immense, yet have rarely been studied [27]. The fact that most first authors of articles resulting from these partnerships are situated in Northern institutions is a source of contention [28]. Our analysis of articles published over the past 30 years on the subject of health funding in Burkina Faso showed that researchers of that country were first authors in only $5 \%$ of the articles on studies in which they were involved [29]. This question takes on increasing importance with the growth of international scientific collaboration. For example, looking at all disciplines combined, the proportion of articles with co-authors from different countries rose from $16 \%$ in 1997 to $25 \%$ in 2012 globally, while, at the same time, the number of authors per article has been steady growing [30]. In the field of natural science and health, $87 \%$ of articles published in 1900 had single authors, but by 2011, that proportion was down to just 7\% [31]. In addition, the question of access to data appears to be very sensitive, as very often the protocols are written in the North with very little involvement of Southern researchers (for many reasons that we cannot go into here for lack of space, see [21]), and then the data are collected by Southern colleagues and analyzed by those in the North. While this description may appear somewhat exaggerated, it is nevertheless a fair representation of what often happens on the ground [20,32].

Moreover, in a context where the time of African researchers is often completely taken up with international consultations [22], it is usually young researchers, students, and assistants who are most closely involved in research processes, data collection and analysis. In the North, scientific collaborations in global health also often involve students (some of whom are also from the South) whose involvement helps to strengthen their research competencies. As such, issues around training up the next generation are added to all the other issues and become crucial [33]. This is all the more 
important, in that ethical challenges are only now starting to be considered in public health doctoral training programs in Canada and are nearly nonexistent in training programs in Africa.

Because of this, many researchers who have been involved in this type of collaboration have occasionally observed misbehaviours, as well as breaches of ethical practice and of data use [34]. Such misconduct is obviously not exclusive to researchers and students working in Africa, but can be found everywhere [35]. Nevertheless, we believe the field of PHIR in a context of global health research collaboration, as described above, can exacerbate such misconduct. What global health researcher has not heard the story of the professor of medicine who, without his partners' knowledge, provided his student with data from an international research project as material for a thesis? Who has never seen, in a list of authors for a scientific article, the name of a researcher who did not contribute in any way to that article's content or writing? Conversely, who has not been surprised to see a researcher who contributed significantly to a study be forgotten in the author list, and even in the acknowledgements? Who has not been astonished that researchers would strive to collect data over many years but then never publish them, or publish too late for the findings to be of use to stakeholders, or expend no effort to make results intelligible for potential users, all without seeing this as an ethical problem?

We could provide numerous pages of examples and try to find reasons to explain them, but that is not the objective of this article. Instead our aim is to advance reflection on issues surrounding researcher conduct and having to do with access to, and use of, data generated by PHIR projects in global health. Thus, our objective in this article is to describe the context and process of developing a policy on the production, access, use and publication of empirical data produced within a global health research program. We believe that sharing our experience of this process, and the content of this policy, will help to stimulate debate among researchers and could potentially be of help to other teams.

\section{Context and process of formulating the internal policy}

The context for this internal policy is that of PHIR, and more specifically PHIR in Africa, within the framework of an international scientific collaboration involving multiple research institutions (public and private) in Germany, Burkina Faso, Canada, and France. This policy was formulated for a research program conducted in Burkina Faso, funded for five years (2011-2016) by CIHR as part of its call for programmatic studies dealing with health equity. In the programmatic research concept, researchers are expected to present a program made up of multiple studies that may evolve over time but that should all converge toward the same objective. In the present case, this objective focused on promoting equity and health by bringing together a group of researchers and decision-makers.

Our programmatic research consisted of a portfolio of PHIR projects to evaluate the impact, effectiveness, and processes of a set of promising community-based interventions to improve equity in Burkina Faso. These interventions had to do with maternal and child health, the health of indigents, febrile illnesses, trauma related to traffic accidents, and health system reforms. To encourage the dissemination and use of results, a knowledge brokering intervention was also formulated, implemented, and evaluated. Several action-research projects were implemented and certain methodological developments were analyzed. Details of this program, its activities and partners are presented elsewhere (see Health Equity in Burkina Faso). The total program budget was CDN\$1.8 million.

The process of formulating this policy unfolded in stages, which we describe below, under the coordination of the principal investigator (PI), who is the first author of this article (VR), with the involvement of certain researchers associated with the program and co-authors of this article. It was undertaken at the end of 2014, around two years after the program's launch. The PI took the lead in producing and coordinating this process, as he had the initiative and the moral responsibility, had 
already studied the issue in previous work, and was the only one with a comprehensive overview of all the PHIR projects included in this program. Nevertheless, the process was inclusive and participative, with everyone having the opportunity to contribute their particular competencies and experiences.

Given the Pl's deep knowledge of the context and of previous analyses of the challenges involved in global health scientific collaboration [20,21], he started this reflection, in a first step, with an analysis of the literature on the topic. A non-systematic review of the literature was done and the results subjected to narrative analysis. The main scientific articles on the topic, including those mentioned above, were studied, along with documents and reports on the responsible conduct of research. However, beyond broad principles and guidelines on research practice, the fact is that very few researchers have published on such processes, and even more scarce are policies accessible on the Internet regarding empirical data. It was therefore necessary, in a second step, to draft an original document that would address the various essential points, inspired largely by principles advanced in key texts from Canada and Quebec on the subject (Box 1). Besides these, numerous other documents from several countries were also studied and are presented in Annex 4 of the policy (Annexe A).

\section{Box 1. Key documents on ethics and the responsible conduct of research}

- The Tri-Agency Open Access Policy on Publications, 2015

- FRQ Policy for the Responsible Conduct of Research, 2014

- Tri-Council Policy Statement: Ethical Conduct for Research Involving Humans (TCPS 2), 2014

- The Tri-Agency (CIHR, NSERC, SSHRC) Framework: Responsible Conduct of Research, 2011

- Le règlement en matière de probité intellectuelle en recherche et développement (Regulation regarding intellectual probity in research and development), CHUM, 2005

These foundational texts are presented in the preamble of the internal policy to ensure that the researchers involved can locate them. The Canadian researchers are expected to already be familiar with them (even though training on this subject is rare), and the others have certainly encountered these principles in similar policies in their countries. However, the aim of the internal policy was primarily to contextualize these guiding principles within an operational procedure specific to our research program. As such, the PI drafted a first version of the internal policy based on all these documents and on the needs of the research program in Burkina Faso.

In a third step, the document was first distributed to a small group of six researchers who were most closely involved with the program and with issues related to research ethics. Two more researchers, who were outside the program (in Canada and in France) but had expertise in this subject and experience working in Africa, were also solicited for informed advice on the draft document. The comments (in the following non-exhaustive list) highlighted the need to:

- explain the issues involved in open access publication of articles;

- explain the concept of data as it is used in the policy;

- take into account the specific nature of qualitative research when talking about the transmission of data and their anonymity;

- $\quad$ propose not only electronic, but also physical, data storage;

- share data cleaning programs used for quantitative data;

- explain the notion of a researcher in the context of a CIHR grant and of multiple research projects within an overarching collaborative research program;

- discuss the congruence between the principles in the internal policy and those of the research ethics committees to which the projects were submitted; 
- distinguish between the people who perform the analyses and those who assist them;

- provide more detail on the issue of inviting those researchers who contributed substantially to the conception of studies to take part in scientific publications.

All of their comments and additions helped to improve the document and to clarify a several points.

In a final step, the second version was sent to the 17 co-researchers in the program to obtain further constructive feedback; a proposal was also made to organize a telephone conference to discuss the content in detail. Only a few additional corrections were requested, and a consensus was reached on the content of the third version. No telephone conference was held, as it was not deemed necessary, showing that there was definitely general agreement on the document. Version four, summarized below (and presented in detail in the Annex) is thus the definitive version adopted by the team; the final policy's content is described in the following section.

\section{Content of the internal policy}

The values embedded in the policy are transparency, prevention, consensus around the production process, compliance with international principles, open access to knowledge produced, and a concern for knowledge dissemination and use. The policy is presented in several sections.

After a general introduction to both the overall and particular contexts within which the policy is positioned, its specific objective is presented, which is "to define rules and procedures specific to this research program with regard to data storage, access, use, and publication, within the framework of these Canadian and Quebec policies and those of the country (i.e. Burkina Faso) in which the studies are being conducted." The term "specific" is underlined to make it clear that this is not a generic policy, but rather one that is tailored to the particular context of this research program. The notion of data is also defined in the policy.

Next, it was essential to specify the status of the different stakeholders in the research program, as three types of researchers are involved, and their statuses have significant implications, particularly with regard to scientific publications and compliance with the rules of the International Committee of Medical Journal Editors (ICMJE) [36]. In our context, there is a principal investigator for the program, and then, for the various research projects, there are lead researchers, researchers in charge of specific studies, and co-researchers collaborating on the different studies. For purposes of transparency, as well as for consensus and to avoid conflicts, a detailed list of all studies conducted in the program and the names of all the researchers associated with these was appended to the policy. This list is updated whenever new studies are added to the program.

The three subsequent sections clarify the rules we adopted regarding i) data storage, ii) data access and use, and iii) scientific production and authorship. On this latter point, we stressed the importance, as stated in the Quebec directives, of "disseminating research results in a responsible and timely manner" [3], as well as the rules of the ICMJE - two subjects with which researchers are generally not very familiar [27].

The policy content is presented in detail in the Annex. It is freely available for use by others and would need to be adapted as appropriate.

\section{Discussion}

Obviously, the process of formulating this internal policy and the decisions regarding its content were not easy. In this discussion we present some of the major challenges encountered. 
Despite a request for support, neither the university nor the research centre with which the PI was affiliated were able to support him in this process; the best they could do was to offer examples of policies from other programs of researchers in these institutions. The research centre provided no examples nor any support, and the few examples provided by the university were not suitable for PHIR and the global health context. This most certainly demonstrates that such approaches remain embryonic in the academic world, which can probably still be characterized as "organized anarchy" [37], as was concluded in the analyses of the 1970s. It seems to us that universities and research centres should be more proactive and provide greater support to their researchers, anticipating ethical challenges. They could, for example, do a better job of sharing experiences and best practices in this area by centralizing and analyzing the different policies and codes of conduct drafted independently by each team or researcher, such that new teams would not need to reinvent the wheel. At the same time, researchers should be more strongly encouraged to produce such internal policies for their own research programs or projects, so as to identify and address as early as possible any potential problems related to data. This will also help ensure that researchers collaborate fairly and respectfully while sharing their best practices and lessons learned.

During the program's first two years, the researchers were fully occupied and focused on preparing and implementing the various research projects and producing data. As such, issues around data use were not considered to be essential in the early days. It is certainly regrettable that the policy was not formulated at the start of the program, as this delay raises concerns about its retroactive applicability. In other programs in which the PI had previously been involved, he had attempted in vain to suggest to the principal investigators that such a policy be drafted. This time, making the most of this first large-scale program under his own responsibility, he spearheaded the formulation of this policy, which he saw as a useful advance. It is never too late to begin a process of reflection in such a program - as was pointed out also by the authors of the Partnership Assessment Toolkit [2].

Most of the program's researchers found the initiative to be original and beneficial, regardless of which continent they were working in. Some of them had encountered most of these issues and appreciated being able to address them calmly in a spirit of team work. However, not all the researchers were equally involved in formulating the policy; some made constructive suggestions, while others did not take part in any of the discussions. These differences in involvement remain to be analyzed. They may signal the pressing need for better training of PHIR researchers in ethical issues pertaining to data [38] and the need for vigilance regarding issues of power and money that can often influence the question of data use in a PHIR context. These are issues that should necessarily be discussed at the start of any collaboration. Based on our experience of international scientific collaborations and of training students in Canada and Africa, we have observed that these challenges are rarely mentioned in public or in team meetings. However, our experience of developing this policy could certainly be adapted to other contexts besides PHIR, notably in any type of scientific partnership, which is often the case in collaborations with community organizations or communities [39].

One unintended positive outcome of this process was that one of the research partners in Burkina Faso decided to formulate its own policy regarding data generated from its population observatory. Even though that observatory had been in place since 2007 [40], and many issues had arisen around data access and use in international scientific collaborations [38,39], no such policy existed. Our policy formulation process had the positive effect of encouraging them to do the same at the end of 2014.

Applying and following up on this policy, however, will continue to be a major challenge. Not only are there questions about how data will be stored and made freely accessible in contexts where our universities have not yet adapted to this new global orientation, but there is also the increasingly pressing issue of who will ensure this follow-up of the policy application, especially after the grant funding comes to an end. Another key issue is what procedures are to be followed in cases where 
someone does not comply with the content of a collectively adopted policy. Additionally, it would be interesting, after this policy has been used and applied for some time, to see how it might be improved and to draw upon our experience to make it even more useful.

\section{Conclusion}

Formulating this type of internal policy regarding data produced by a research program should definitely be viewed more as an act of prevention than one of sanction. Trust, transparency and mutual respect should ultimately be core elements of every scientific partnership in the fields of health and of interventional research in general [2], but an ounce of prevention is definitely worth a pound of cure... Hygieia and Panacea [43]!

\section{References}

\section{[See Références]}




\section{INTERNAL POLICY REGARDING STORAGE, ACCESS, USE, AND PUBLICATION OF DATA}

\section{Introduction}

This research program is essentially focused on community-based interventions aimed at addressing the most significant health and health equity problems in the country, as well as those problems that are most neglected. The goal of the program is to study the effectiveness and processes of promising interventions for improving health equity in Burkina Faso. The program's principal investigator is Valéry Ridde, and the program has been funded by CIHR for the period 2011-2016.

Within the framework of this program, numerous studies have been conducted (see Annex 1) and several others will be undertaken between now and the end of the funding period. As such, a great deal of quantitative and qualitative data has been collected. Given that there are many researchers involved in this program, as well as students and research professionals (assistants, engineers, etc.), it is essential that a clear, fair, and transparent policy be defined with regard to the management and use of these data.

This policy, which is internal to the research program, is not intended to replace the rules and best practices (Annex 4) already in place, with which the researchers must necessarily be familiar. In essence, everyone involved in this research program is expected to respect the existing rules regarding ethics and responsible conduct of research, particularly:

- $\quad$ The Tri-Agency Open Access Policy on Publications, 2015

- The Politique sur la conduite responsable en recherche des FRQ (FRQ policy for the responsible conduct of research), 2014

- The 2nd edition of the Tri-Council Policy Statement: Ethical Conduct for Research Involving Humans (TCPS 2), 2012

- The Tri-Agency Framework: Responsible Conduct of Research, 2011

- The Règlement en matière de probité intellectuelle en recherche et développement (Regulation regarding intellectual probity in research and development), CHUM, 2005

\section{Purpose}

The purpose of this document is to define rules and procedures specific to this research program with regard to data storage, access, use, and publication, within the framework of these Canadian and Quebec policies and those of the country in which the studies are being conducted (i.e., Burkina Faso).

In this policy, the concept of data is defined as any information collected or used in the program's research activities that is intended to be compiled, or is already compiled, in data banks created for that purpose. This information includes responses provided by individuals to quantitative questionnaires, as well as the results of tests performed, biological specimens collected, geographic coordinates, information drawn from administrative records, and the content of formal qualitative interviews. While documentary analyses, notes taken, and even log books are considered to be useful data in qualitative research, they are not considered to be data for the purposes of this policy.

\section{Typology of researchers}

This internal policy applies to the research program and all projects for which protocols have been submitted to the research ethics committees in both countries. In addition to the program's principal investigator, each protocol specifies the existence of three types of researchers: lead researchers, researchers in charge of specific studies, and co-researchers collaborating on the different studies. The names of these researchers are presented in Annex 1. This list will be updated whenever any new research projects are submitted between now and the end of the funding period. Using Annex 5, researchers will be able to obtain from their collaborators signed commitments that they will respect this policy. 


\section{Data storage}

1. All data derived from studies ${ }^{2}$ (Annex 1) must be systematically sent by the researcher in charge of each study to the research program coordinator when data collection is completed.

2. All original data will be stored by the program coordinator, who will ensure they are stored either on the server (CHUM or University of Montreal) or on paper, in a secure location.

3. Researchers in charge of studies will keep a copy of the original data for subsequent analyses.

4. All data must be anonymized and securely stored, with protected access.

5. All data must be stored for a minimum of 5 years after the end of the grant funding period.

6. No data may be destroyed without the consent of the research program's principal investigator, and the principal investigator may not destroy any data without the consent of the researchers in charge of those studies.

7. The data cleaning program used for quantitative data must be transmitted to the program coordinator once the work has been completed.

\section{Data access and use}

8. No data may be transmitted to any persons (students, statisticians, translators, assistants involved in data cleaning or transcription, researchers, etc.) who are not identified in the research protocols submitted to the ethics committees (Annex 1).

9. Transmission of data to other persons for purposes of analysis is possible, but only if the analysis is performed under the supervision of a researcher identified in one of the protocols (Annex 1).

10. Any researcher on the team (Annex 1) who wishes to engage another person and provide him/her with access to these data must obtain a written, signed request from that person (Annex 2). This form must be sent to all researchers in charge of every study (Annex 1) or, in their absence, to the lead researcher(s), using the template provided in Annex 2. Researchers who take on the responsibility of supervising this analysis work must counter-sign the thirdparty request for access to data, in order for it to be accepted. This document must then be sent, for information purposes, to the program coordinator.

11. All data transmission must protect the anonymity and privacy of research participants.

12. Persons who are not identified in the research protocols submitted to the ethics committees (Annex 1) and who are subsequently authorized to access the data must agree to respect the present policy.

13. A deliberative process will be undertaken over the coming months around making the research program data freely available to outside researchers, since for the time being, data transmission and analysis are only possible under the supervision of each researcher in charge of a study or the lead researcher.

14. In cases where these internal rules of the present policy regarding data storage, access, and use are not presented in the protocols submitted to the different ethics committees, the lead researchers will submit requests for amendment.

\section{Scientific productions and authorship}

15. Researchers who lead studies or participate in any of the research program's various projects are strongly encouraged to publish the results of their research for the benefit of the scientific community and the population at large. The FRQ considers that responsible conduct involves

${ }^{2}$ Longitudinal data collected by the IRSS population observatory (known as Kadess), including those funded over one year by the Equity program, are not subject to this policy but instead are covered under its code of use dated December 22, 2014. 
"disseminating research results in a responsible and timely manner".

16. The rules regarding authorship of scientific productions in the context of the research program are defined as follows and are aligned with the precepts of the International Committee of Medical Journal Editors (2013).

17. The intellectual process leading to a scientific production (article, poster, oral communication, book chapter, policy brief, etc.) includes the following stages:

i. Making a substantial contribution to the conception OR design of the study, OR to the data collection and analysis, OR to interpretation of the data; AND

ii. Drafting a first version of the publication OR re-reading and revising that version, making a critical intellectual contribution; AND

iii. Approving the final version for publication; AND

iv. Assuming responsibility for guaranteeing all aspects of the work, and in particular, the accuracy OR integrity of the content of the article AND of the relevance of the study and analyses.

18. All persons designated as authors in a scientific production must satisfy these four criteria, and anyone who satisfies these four criteria must be named as authors. Those who do not satisfy these criteria but contributed to the study should be named in the acknowledgements section of the production.

19. These criteria are not to be used to deny anyone the right to authorship of a scientific production, either by not involving them or not giving them the opportunity to be associated with stages 2 (ii) and 3 (iii). As such, anyone who has taken part in the first stage (i), thereby satisfying the first criterion, must necessarily be given the opportunity to be an author of any resulting scientific productions, particularly by playing a role in the writing, revision and final approval. In the context of the research program, this means that all lead researchers and all researchers in charge of the different studies (Annex 1) must have the opportunity to participate in scientific productions. If other researchers outside of these two categories were involved in the first stage, they should also be given this opportunity. Not making this opportunity available is considered a breach of best practice in the health field.

20. To ensure full transparency in this process, every person who decides to undertake a scientific production must therefore inform all parties whom he/she wishes to involve in that production. This proposal should be made in accordance with the plan set out in Annex 2 (article proposal). Everyone who is contacted will be given 10 working days to respond to this opportunity. Anyone failing to respond within the allotted time will be considered as having declined to participate in this scientific production. Once agreement has been reached among the authors, all article proposals must be sent to the research coordinator for information purposes. This preliminary agreement does not necessarily guarantee every person the status of author, as this status will depend on their satisfying the four criteria (item 17 above), such that this agreement is subject to revision at any time in accordance with these rules.

21. Authorship order should be decided by all the co-authors in a transparent manner and should reflect actual contributions throughout the writing process. The first author is the one who has taken the lead in writing the article; that person contributes the most and is responsible for the scientific content (particularly with respect to avoiding potential plagiarism, errors, etc.) and for contact with co-authors. Authors cannot be named if they have not given their approval for the document.

22. Researchers are expected to make every effort to ensure all their scientific productions are available in open access. CIHR policy stipulates that, for scientific articles, researchers must arrange to publish in journals that provide open access either immediately or within 12 months of publication. The research program is prepared, within the limitations of its available budget, to cover article processing charges (APC). If the researchers do not wish to publish in such a journal, they must ensure that the selected journal allows for articles to be placed in a central 
or institutional repository in the 12 months following publication.

23. Some journals require that the databases and data collection instruments upon which publications are based be made accessible on the Internet. In such cases, the first author of the article must communicate with the research program coordinator before any publication to find a suitable technical solution.

24. Copies of all scientific productions (article, poster, oral communication, book chapter, policy brief, use in course curriculum, etc.) based on data derived from research projects must systematically be sent to the research program coordinator for information after being presented. Scientific productions will be posted on the research program website (www.equitesante.org) with the consent of the researchers responsible for their preparation. With a view to sharing knowledge, researchers should make every effort to ensure most of their work is posted on the website, recognizing that this may not always be possible or desirable (for example, in the case of preliminary data analyses).

25. All scientific productions must acknowledge the CIHR funding using the following sentence: "This research project is part of the 'Community research studies and interventions for health equity in Burkina Faso'. We thank the Canadian Institutes of Health Research (CIHR) who funded the program (Grant number ROHU115213)."

26. All conflicts of interest for every author associated with the scientific production must be presented.

\section{Failure to respect the provisions of this policy}

27. Anyone who is aware of any failure to respect the provisions of this policy must immediately inform the principal investigator of the program.

28. In cases where such failure is observed, the principal investigator of the program will endeavor to understand and resolve the situation amicably with the individuals concerned and, if necessary, with the program's committee of co-investigators.

29. In any case where such amicable resolution is not possible, the principal investigator will inform the university of the observed failure.

[For Annexes 1-5, see Annexe A] 